\title{
Zero Pronouns and Conditionals in Japanese Instruction Manuals
}

\author{
Tatsunori Mori and Hiroshi Nakagawa \\ Division of Lilectrical and Computer Enginecring, Faculty of Engineering, Yokohama National University, \\ Tokiwa-dai 156, Ilodogaya-ku, Yokohama 240, JAPAN \\ E-mail: mori@forest.dnj.ynu.ac.jp, nakagawa@naklab.dnj.ynu.ac.jp
}

\begin{abstract}
This paper proposes a method of the zero pronoun resolution, which is one of the essential processes in understanding systems for Japanese manual sentences. It is based on pragmatic properties of Japanese conditionals. We examined a number of sentences appearing in Japanese manuals according to the classification based on the types of agent and the types of verb phrase. As a result, we obtained the following pattern of usage in matrix clauses: 1) The connective particles TO and REBA have the same distribution of usage. TARA and NARA have the same distribution of usage. 2) The distribution of usage of TO and REBA, and that of TARA and NARA are complementary to each other. We show that these distributions of usage can be used for resolution of zero subjects.
\end{abstract}

\section{Introduction}

From simple electrical appliances to complex computer systems, almost all machines are accompanied by instruction manuals. Since recently there are many machines whose operating procedures are complicated, we have much trouble in many cases including translating their manuals into other languages, maintaining consistency between the description in manuals and the actual behavior of the machines. To solve these problems, we have to have a computer assisted system for processing Japanese manual sentences, especially for understanding manual sentences.

$\Lambda$ large number of researchers have gotten to grip with the method of understanding some types of text including instruction manuals(Abe et al., 1988; Nomura, 1992; Eugenio, 1992). One of the most important matters of concern in these types of system is how we can fix ambiguities in semantic representations and fill underspecified parts of them. Generally speaking, almost all systems described above take the following scheme. Firstly, each sentence in' a text is translated into a semantic representation. In this process, the system uses only non-defeasible syntactic and semantic constraints. Most of pragmatic information and commonsense knowledge are not used here, because the result of these knowledge would be overrid- den by some other information such as contextual information. Therefore the semantic representation would include some undetermined parts which would be fixed by other kind of information including context. This way of analysis is known as the Nondefeasibility Thesis(Kameyama, 1995). Secondly, all of undetermined parts of the semantic representation are filled or settled by some kind of inferences based on the domain knowledge.

This type of method, which uses a large amount of domain knowledge, seems to be dominant from the vicwpoint of disambiguation. Moreover it scarcely depends on the language in use because the way of disambiguation is based on the inference with a certain knowledge base. On the other hand, in order to use this method, we have to prepare the amount of knowledge being large enough to cope with various type of described objects. Unfortunately, so far we have not had such a commonsense knowledgo base.

One of ways to get rid of this situation is to adopt some knowledge which hardly depends on some particular domain. As such a kind of knowledge, we pay our attention to pragmatic constraints, which have not been used sufficiently in the former methods. We expect that by pragmatic constraints the ambiguity in manual senttences would be resolved to some extent not in the process of inference but in the process of tho translation of manual sentences into semantic representations.

We do not commit ourselves to the domain specific knowledge, but use some ontological knowledge in general manuals. For example, the correspondence of objects in the manual sentences to the objects in linguistic constraints, like the speaker, the hearer, and so on. Note that the ontology in this paper does not refer to all of objects in the world described by manuals, like a certain part of machine. Aiming at independence from the domain knowledge of objects, we adopt one of general ontologies which is applicable to almost all manuals. In short, our scheme consists of the following three parts: 1) a parser based on the nondefeasiblity thesis, 2) pragmatic constraints specific to linguistic expressions, and 3) the general ontology of the world described by manuals.

In the rest of this paper, we will focus on the zero pronoun resolution. In Japancse, zero pronouns frequently make a sentcnce ambiguous. Zero pronouns are ellipsis of obligatory cases, which very frequently appear in Japanese sen- 
tences. Pispecially, subjects are omitted very often. It is called "zero subject." In some sense, the resolution of zero pronouns' referents, especially the resolution of "zero subject", is the essential part of the knowledge extraction from Japanese manuals, because once referents of zero pronouns are identified, we can use various methods already been proposed to recognize the structure of sentence and to map it into the suitable knowledgo representalion. To capture pragmatic constraints, we have paid our attention to conditionals, which occur very frequently in instruction manuals. In this paper, we will show that in instruction manuals, the constraint of conditionals can be used to identify the referents of zero subjects. Although, of course, not all the zero pronomins can be solved with the constraints shown in the paper, our $\mathrm{cx}$ amination for a lot of manual sentences shows that the constraints work very effectively and accurately in sentences with conditionals.

Now we have to define the term 'subject' we used in this paper. Generally, the term 'subject' denotes a nominative from the grammatical point of view. In this paper, however, we will use the term SUBJECT to denote a main participant of tho sentence. Roughly speaking, in the active voice, the SUBJECT is the nominative, on the other hand, in the passive voice, the SUBJECT is the nominative of the corresponding sentence in the active voice.

\section{Zero pronouns in manual sentences}

Let's consider the following Japanese sentence, which shows a certain instruction.

(1) $\phi_{a}$ kono-botan-o osu -to,

$\phi_{a}$-NOM this-butiton- $\Lambda \mathrm{CC}$ push -TO

$\phi_{b} \quad$ der -are -mas -u.

$\phi_{b}$-NOM go out -can-POL -NONPAST.

If $\phi_{a}$ push(es) this button, then $\phi_{b}$ can go out.

Native speakers of Japanese liave the following intuitive interpretation for (1) without any special context.

(2) $\phi_{a}=\phi_{b}=$ the hearer (= the user)

Here, 'TO' is a Japanese conjunctive particle which represents a causal relation. 'MASU' shows politeness, which is expressed by poL in (1). 'The 'ARE' shows ability or permission.

On the other hand, the following sentence, which does not have the verbal suffix of possibility 'ARE' in the matrix clause, has a different interpretation.

(3) $\phi_{c}$ kono-botan-o osu -to, $\phi_{c^{-N O M}}$ this-button-ACC push -TO, $\phi_{d} \quad$ de - mas $-\mathrm{u}$.

$\phi_{d}$-NOM come out -POI -NONPAST. ${ }^{1}$

If $\phi_{c}$ push(es) this button, then $\phi_{d}$ will come out.
The zero pronoun $\phi_{d}$ does not refer to the hearer(the user), even though $\phi_{c}$ refers to the user as well as (1). 'The intuition of native speakers of Japanese for (3) is that $\phi_{d}$ refers to a machine or a certain part of the machine. Note that when only the matrix clause of (3) is used as shown in (4), $\phi_{e}$ can be interpreted as either the hearer or the machine $^{2}$

(4) $\phi_{e}$ de -mas -u.

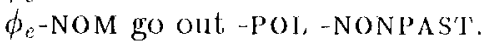

$\phi_{e}$ will go out.

These examples show that the expressions To and ARE impose some constraints on the referents of SUBJECTS of the sentences. As described above, there are many cases that linguistic expressions give us a key information to resolve some type of ambiguity like the anaphora of a zero pronoun. In the rest of this paper, we will show several pragmatic constraints, which can account for the in terpretations of these sentences described above.

Dohsaka(Dohsaka, 1994) proposes a sirrilar approach, in which pragmatic constraints are used to determine referents of zero pronouns. While his approach treats dialogue, our targets are manwal sentences. His approach utilizes honorific expressions and the speaker's point of view. Since the constraints are effective in the different target from ours, the accuracy of identifying the referents of zero pronouns would be improved much more by using both of his constraints and the constraint we proposed. As for the identifying method available in general discourses, the centering theory(Brennan et al., 1987; Walker et al., 1990) and the property sharing theory(Kameyama, 1988) are proposed. Although this kind of theory has a good point that it is independent of the type of discourse, the linguistic constraints specific to expressions like the pragmatic constraints proposed by Dohsaka or us are more accurate than theirs when the specific constraints are applicable.

\section{General ontology in manuals and primary constraints}

In this section, we consider the general ontology which can be used in all types of manuals.

We should consider two types of information as the parts of ontology: the properties of the objects in manuals and the discourse situation that is characterized by linguistic roles like a writer and a reader.

\section{Constraint 1 (Objects)

User has intention. \\ Manufacturer has intention. \\ Machine has no intention.}

'The Finglish translation of 'DERU' in (3) is dif ferent from the translation in (1). It is due to the difference of the vicwpoint between Japanese and English. The difference has no effect on the selection of zero pronoun's referent.

${ }^{2}$ It seems to be more natural that $\phi_{e}$ is interpreted as the hearer. 
Constraint 2 (Discourse Situation) Speaker(Writer) $=$ Manufacturer Hearer(Reader) $=U$ ser

From these constraints of the ontology, we can obtain the constraint of persons as follows.

Constraint 3 (Persons)

First Person $=$ Manufacturer
Second Person $=$ User
Third Person $=$ Machine

Before considering the constraints of Japancse conditionals, we had better mention the more basic expressions in manuals. In Japanese, simple operation procedures, like those which do not include some conditions, are often described as simple sentences with no subjects whose verbs are of one of the following types: the RU form, the request form or the solicitation form. The RU form is the basic form of verbs and it denotes the non-past tense. Since the RU form has a neutral meaning, it does not impose any restriction on the SUBJECT.

On the other hand, the request form and the solicitation form have some constraints. The speaker uses the sentences to prompt hearers to do an action described by the sentence. Therefore, we have the following constraint.

Constraint 4 (SUBJECT of sentence in the request form)

The SUBJECT of a sentence in either the request form or the solicitation form is the hearer.

Manual sentences may have a kind of modality expressing the permission, the possibility, the obligation, and so on. Sentences which have the expressions of ability or permission mean not only that it is possible for the SUBJECT of the sentence to do the action, but also that the SUBJECT has their choice of whether to do the action or not to do it. Therefore, we have the following.

Constraint 5 (SUBJECT of sentence with ability expressions)

$A$ SUBJECT of a sentence with the expressions of ability or permission must have his/her intention to make a choice about the action described by the sentence.

\section{Semantics of Japanese Conditionals}

Japanese has four conditional particles, TO, REBA, TARA and NARA, which are attached to the end of subordinate clauses as described in (1). The subordinate clause and the matrix clause conjoined by one of these particles correspond to the antecedent and the consequence, respectively. Each expression has its own meaning as shown in Table 1(Masuoka, 1993). TARA and NARA are very rarely used in manual sentences as far as we examined. For example, the rates of use of each conditional in over a dozen of instruction manuals are as follows ${ }^{3}$ : TO is $77.6 \%$ ( 385 sentences) of all conditionals, REBA is $19.4 \%$ (96 sentences), TARA is 2.6

\footnotetext{
${ }^{3}$ As described later, we have examined several other manuals especially for the consideration of the
}

$\%$ (13 sentences) and NARA is $0.4 \%$ ( 2 sentences). Roughly speaking, TO and REBA show causality relations, namely some general rules, and TARA and NARA are used in the case that the the antecedent is an assumption. The fact that not assumptions but general rules are usually described in the context of instruction is the reason why TARA and NARA are used less frequently than TO and REBA.

The difference of constraints of these expressions are shown in the following sentences, which are the variants of the sentence (3).

(5) $\begin{array}{ll}\phi_{i} & \text { kono-botan-o ose -ba, } \\ \phi_{i} \text {-Nom this-button-ACC push -REBA, } \\ \phi_{j} \text { de -mas - }\end{array}$ $\phi_{j}$-NOM come out -POL -NONPAST. If $\phi_{i}$ push(es) this button, then $\phi_{j}$ will come out.

(6) $\phi_{k} \quad$ kono-botan-o osi -tara, $\phi_{k}$-NOM this-button-ACC push -TARA, $\phi_{l}$ de -mas -u. $\phi_{1}$-NOM come out/go out-POL -NONPAST. If $\phi_{k}$ push(es) this button, then $\phi_{l}$ will come out/go out.

(7) $\phi_{m}$ kono-botan-o osu nara, $\phi_{m}$-NOM this-button-ACC push -NARA, $\phi_{n}$ de -mas $-\mathrm{u}$.

$\phi_{n}$-NOM come out/go out -POL -NONPAST.

If $\phi_{m}$ push(es) this button, then $\phi_{n}$ will come out/go out.

As well as the sentence (3), for Japanese native speakers, the SUBJECT of the matrix clause of (5) should be a machine. On the other hand, in the case of the sentences (6) and (7), the SUBJECTS of the matrix clauses can be either users or machines. These phenomena probably due to the nature of each conditionals. Since a causal relation, which is shown by TO or REBA, expresses a general rule, the consequence cannot include speaker's attitude, like volition and request. Therefore, the SUBJECT of the matrix clause should be a machine. In contrast, in the case of assumptions, that is TARA and NARA, there are no such restrictions on the SUBJECT.

It depends on the volitionality of the verb whether a sentence shows a speaker's attitude, or not. Therefore, we consider each Japanese conditionals in terms of volitionality of the verb. Note that the electronic dictinary IPAL provides the information of volitionality for each Japanese verb entry(IPA Technology center, 1987). We can use it to analyze sentences based on our proposal.

\subsection{SUBJECTS of complex sentences with the conditional TO}

A matrix clause of the sentence with TO expresses a consequence of a causal relation. Consequentily,

conditionals REBA, TARA and NARA, since they occur less frequently than TO in manuals and we have to collect more examples to estimate their property in manuals. 
lable 1: Characteristics of Japanese Conditionals

\begin{tabular}{|cl|}
\hline TO & shows successiveness of two events observed in a real situation. \\
\hline REBA & $\begin{array}{l}\text { shows a universal causal relation. } \\
\text { It becomes more assumptive when the subordinate clause shows a state. }\end{array}$ \\
\hline TARA & $\begin{array}{l}\text { shows 1) two individual events occur with the passing of the time, or } \\
\text { 2) an event which is expected to occur on the uncertain assumption expressed } \\
\text { in the subordinate clause. }\end{array}$ \\
\hline NARA & $\begin{array}{l}\text { shows that the antecedent of the sentence is an assumption } \\
\text { and the consequence holds on that assumption. }\end{array}$ \\
\hline
\end{tabular}

in matrix clauses, we can use either the mood of the description of facts or the mood of evidentials like conjectures, judgment, and so on. In contrast, we may not use the expressions of volition, requests and so on. We consider only the mood of the description of facts, because mamual sentences should describe only lacts and must not include speaker's attitude. 'The sentences having; the mood are classified into two types: the description of an action and the description of a state like an expression for the ability of some action. The former type is problematic, because the RUform, which is the normal inflection form of verbs and describes an action, is ambiguous in its meaning. The RU-form can show one of the followings: speaker's volition, speaker's request, to hearers, or the action done by a third party.

In the analysis of the description of an action, it is important to examine whether the verb phrase expresses a volitional action or not. According to the classification by $\mathrm{IP}^{\prime} \Lambda\left(\mathrm{Il}^{\prime} \Lambda\right.$ 'Iechnology center, 1987), all of Japanese verbs are classified into two types, volitional verbs, which usually express intentional actions, and non-volitional verbs, which express non-intentional actions. Although non-volitional verbs only express non-volitional actions, volitional verbs are classified into two kind of verbs. One is the type of verbs which can be used for not only volitional actions but also non-volitional actions. 'The other is the type of verbs which are used only for volitional actions. For example, ITAMU(have a pain) is a non-volitional verb, OTOSU (drop/loose) is a volitional verb which has also the non-volitional use, SAGASU (scarch) is a volitional verb which has only the volitional usc.

Let us consider the interpretations of the matrix clauses of the sentences with To. The first case is that verbs in the matrix clauses are in volitional use. If the SUBJECT is the speaker, the verb in volitional use expresses speaker's volition. If the SUBJECT is the hearer, the speaker expresses his/her expectation that the hearer makes a volitional action shown by the sentence. This is the case of requests. Consequently, the SUBJECT should be neither the speaker nor the hearer due to the constraint that we cannot express some volition or request in a matrix clause of the TO sentence. On the other hand, a third party can be the SUBJECT, because a sentence whose SUBJECT is a third party does not express any volition, in- vitations, requests or injunctions. Since the manufacture is the speaker and the user is the hearer according to the constraint of the discourse situation, the manufacture and the user camnot be the SUBJECT of the matrix clause. Therefore, the only possible interpretation is that the SUBJECT of the matrix clanse is the machine.

'The second case is that verbs in the matrix clauses are in non-volitional use. If a verb of the matrix clause has a non-volitional use, that is, if it is possible for the action of the clause to be done unconsciously, the constraint is not applied, because the verb in non-volitional use does not express any volition, invitations, requests and injunctions. For example, the SUBJECT of the matrix clause of the following sentence refers to the users.

(8) $\phi_{j} \quad \phi_{g} \quad$ fureru-to, $\phi_{f}$-NOM $\phi_{y}-\Lambda$ CO tonch-TO, $\phi_{h} \quad$ kandenshi-nas-u. $\phi_{h}$-NOM get_an_electric_slıock-POL-NONI'ASI. II $\phi_{f}$ touch(cs) $\phi_{g}$, then $\phi_{h}$ will get an elcetric shock.

'To examine the accuracy of interpretations based on our estimation we have collected about, 400 sentences, which include TO and some of which also include possibility expressions, from several types of manuals. By these sentences, we check Constraint, 5 and our estimation of TO. Then, it is confirmed that there are no exception to them, at least in the collected sentences.

\subsection{SUBJECTS of complex sentences with the conditionals REBA, TARA and NARA}

Because of the characteristics of each conditionals described in 'lable 1, we expect that a) the conjunctive REBA, which shows a causal relation, has the same constraint as TO has, which also express causality, b) since both of TARA and NARA express an assumption, they have the same type of constraint, which is diflerent from the constraint, of TO and REBA. As the first step to confirm this expectation, let us exarnine whether the matrix clause may have a request form, or not, in the cases of REBA,TARA and NARA. At first, note that the hearer, namely the user, is the agent of the requested action if the matrix clause is a request. form. In the case that the conjunctive shows causality, the matrix clause should show some inevitable result of the event expressed by the 
subordinate clause. 'Iherefore, the matrix clause should not express the judgement and attitude of the speaker. As for the conjunctive REBA, the fact that the conjunctive represents some causality means that the matrix clause does not have a request form. Note that the exception is the case that the subordinate clause is stative or a non-volitional action. As described in 'Iable 1, in those cases, the subordinate clause shows an assumption rather than a cause, and the matrix clause may be a request as shown in the following example.

(9) hitsuyou-ga nake -reba,

Necessity-NOM there-is-no -REBA,

$\phi_{o} \quad \phi_{p} \quad$ sutete -kudasa -i.

$\phi_{o}$-NOM $\phi_{p}$-ACC discard -REQPOL -NONPAST.

If there is no need of $\phi_{p}$, please discard $\phi_{p}$.

The usages of the conjunctives TARA and NARA which express assumptions, are explained as follows. Since the assumptions are introduced by the speaker, the matrix clause is to describe speaker's expectation or desire. Therefore, it is quite probable that not only the normal form but also some request form, which is considered as a kind of wish, appears in the matrix clause.

In order to ascertain our estimation, we have examined a bunch of real sentences, which appear in real instruction manuals. First of all, in about 400 TO sentences, all of the matrix clauses have no request form. In the REBA case, few request form appear in the matrix clauses. The exceptions are the same type of sentences as (9).

Next, we consider the usage of TARA and NARA. Even if the conjunctive REBA in the sentence (9) is changed for TARA or NARA, the sentences are still acceptable. As we exepcted, it shows that the matrix clause of the sentence with TARA or NARA may have a request form, that is, the SUBJECT of the matrix clause may be a user. Then, can the SUBJECT of the matrix clause be a machine? We expect that there are few cases that the SUBJECT of the matrix clause is a machine, because the highly context specific assumption, which is expressed by TARA or NARA, is not suitable for the description of general rules. Moreover, from the fact that the matrix clause of TO and REBA cannot express the speaker's attitude, we pragmatically infer that TARA and NARA are expected to be used only for expressing the speaker's attitude. Our expectation is summarized in Table 2. Note that a SUBJECT should be either a user or a machine because manufacturers have finished all the actions appeared in the context of instruction before shipment.

Our estimation about To has been already confirmed in Section 4.1. In order to confirm our estimation about REBA,TARA and NARA, let us examine real examples. Since the constraints we pursue here are those which restrict the types of SUBJECTS, we examined the correlation among the types of conjunctives, the types of verbs and the SUBJECT. As for the types of SUBJECTS, a SUBJECT should be either a user or a machine.
Table 2: Our estimate of the usage of the matrix clause

\begin{tabular}{|l|l|l|}
\hline & $\begin{array}{l}\text { Speaker's attitude }= \\
\text { User's Volitional Act. }\end{array}$ & Others \\
\hline TO,REBA & Not avallable & Avalable \\
\hline TARA,NARA & Avallable & Not avalable \\
\hline
\end{tabular}

As for the types of verbs, each clause is classified into two classes according to volitionality of verb. One of them is the class of verbs in volitional use, the other is the class of other non-volitional predicates. Therefore each clause belongs to onc of the followings:

- SUBJECT $=$ user and Predicate $=$ verb in volitional use (U/V, hereafter)

- SUBJECT $=$ user and Predicate $=$ others $(\mathrm{U} / \mathrm{O})$

- SUBJECT $=$ machine and Predicate $=$ verb in volitional use $(\mathrm{M} / \mathrm{V})$

- SUBJECT $=$ machine and Predicate $=$ others $(\mathrm{M} / \mathrm{O})$

Table 3,4 and 5 show the distribution of usage of each conjunctive. Each number shows the frequency of use in the examples we examined. Note that to create 'Table 3,4 and 5 , several Japanese native speakers determine referents of zero SUB JECTS according to contexts.

Table 3: Distribution of use of REBA

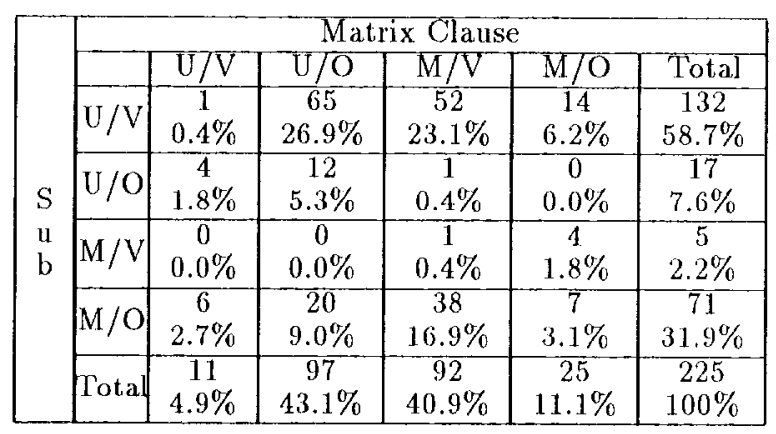

First of all, as we expected before, the distribution of the use of REBA is different from those of TARA and NARA. While we can see several differences of use, the most remarkable one is the difference of use of the matrix clause. The matrix clauses of REBA are hardly any user's volitional action. The exceptions are only about $5 \%$ of all examples. 'The distribution of use of the matrix clauses of TARA and NARA is complementary to the distribution of REBA, that is, the majority of the matrix clause of TARA(about $90 \%$ of all examples) and NARA(100\% of all examples) are user's volitional actions, although the number of the total examples of NARA is not so numerous. The empirical result supports the our estimation. 
Table 4: Distribution of use of TARA

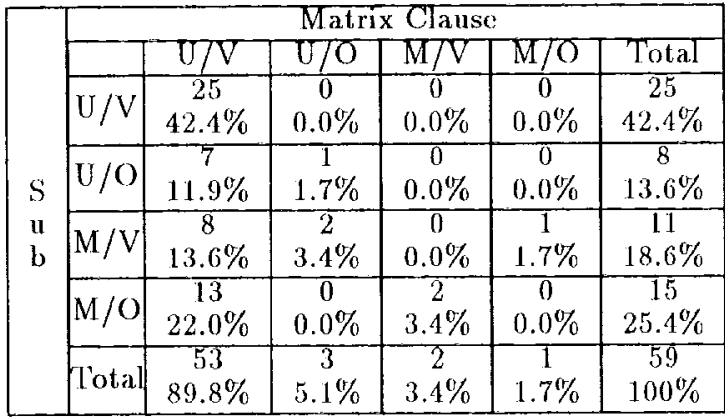

Table 5: Distribution of use of NARA

\begin{tabular}{|c|c|c|c|c|c|c|}
\hline & \multicolumn{6}{|c|}{ Matrix Clause } \\
\hline \multirow{10}{*}{$\begin{array}{l}\text { S } \\
\text { u } \\
\text { b }\end{array}$} & & $0 / \mathrm{V}$ & $0 / 0$ & $\mathrm{M} / \mathrm{V}$ & $\mathrm{M} / \mathrm{O}$ & Total \\
\hline & $\mathrm{J} / \mathrm{Vl}$ & 0 & 0 & 0 & 0 & 0 \\
\hline & $0 / 2$ & $0.0 \%$ & $0.0 \%$ & $0.0 \%$ & $0.0 \%$ & $0.0 \%$ \\
\hline & $0 / 0$ & $\begin{array}{c}8 \\
88.9 \%\end{array}$ & $\begin{array}{c}0 \\
0.0 \%\end{array}$ & $\begin{array}{c}0 \\
0.0 \%\end{array}$ & $0.0 \%$ & $\begin{array}{c}8 \\
889 \%\end{array}$ \\
\hline & & 0 & 0 & 0 & 0 & 0 \\
\hline & $M / V$ & $0.0 \%$ & $0.0 \%$ & $0.0 \%$ & $0.0 \%$ & $0.0 \%$ \\
\hline & $\mathrm{M} / \mathrm{O}$ & 1 & 0 & 0 & 0 & 1 \\
\hline & $101 / 0$ & $11.1 \%$ & $0.0 \%$ & $0.0 \%$ & $0.0 \%$ & $11.1 \%$ \\
\hline & Total & $\begin{array}{c}9 \\
100 \%\end{array}$ & $\begin{array}{c}0 \\
0.0 \%\end{array}$ & $\begin{array}{c}0 \\
0.0 \%\end{array}$ & $\begin{array}{c}0 \\
0.0 \%\end{array}$ & $\begin{array}{c}9 \\
100 \%\end{array}$ \\
\hline & & & $0.0 \%$ & $0.0 \%$ & $0.0 \%$ & $100 \%$ \\
\hline
\end{tabular}

\subsection{Default rules of usage of REBA, TARA and NARA}

The tendency of use of the conjunctives gives us a couple of strong defaults to resolve the zero pronoun in the matrix clauses. We propose the following defaults.

Default 1 (SUBJECT of sentence with TO or REBA)

In a complex sentence with the connective particle TO or REBA, the matrix clause does not express user's volitional action. Therefore, the SUBJECT of the matrix clause should be a machine, if the verb of the matrix clause does not have the nonvolitional use.

\section{Default 2 (SUBJECT of sentence with TARA} or NARA)

In a complex sentence with the connective particle TARA or NARA, the matrix clause expresses only user's volitional action. Therefore, the SUBJECT of the matrix clause should be a user.

The accuracy of the default rules of TO,REBA,TARA and NARA is $100 \%, 95.1 \%, 89.8 \%$ and $100 \%$, respectively, as far as we examined.

\section{Conclusion}

In this paper, we proposed a scherne which closely depends not on domain knowledge of objects described in manual but on pragmatic constraints which linguistic expressions innately have. This method uses only the linguistic constraints and the general ontology of the world described by manuals. Especially, we have shown that we can determine the referents of zoro pronouns to some extent with our linguistic constraints, like the constraint of the Japanese Conditionals. However, we do not have enough knowledge about the following points. They are important portions of our future work.

- Utilization of discourse structure.

- Analysis for the other types of manual scntences, like definitions.

\section{References}

Norihiro $\Lambda$ be, 'Tomohiro Ishikawa, and 'Tsuji Saburo. 1988. Generating an assembly procedure from assembly instruction. Journal of Japanese Society for Artificial Intelligence, 3(5):590-598, Septernber. (in Japanese).

Susan E. Brennan, Marilyn Walker Friedman, and Carl J. Pollard. 1987. A centering approach to pronouns. In Proceedings of the 25th Annual Meeting of the American Association of CompuLational Linguistics (ACL 87), pages 155-162.

Koliji Dohsaka. 1994. Identifying the referents of Japanese zero pronouns based on pragrnatic condition interpretation. Transactions of Informalion Processing Society of Japan, 35(5):768 778. (in Japancse).

Barbara Di Eugenio. 1992. Understanding natural language instructions: The case of purpose clausc. In Proceedings of $301 h$ Annual Meeting of $A C l$, pages $120-127$.

IPA 'Technology center, 1987. The lextcon of the Japanese basic verbs for Computers. Information-technology Promotion Agency $(\mathrm{IP} \Lambda)$, Japan, March. (in Japancse).

Megumi Kameyama. 1988. Japanese zero pronominal binding: Where syntax and discourse meet. In W. Poser, cditor, Japanese Synlax, pages 351-369. CSLI, Stanford.

Megumi Kameyama. 1995. 'The syntax and semantics of the Japanese language engine. In R. Mazuka and N. Nagai, editors, Japanese Syntactic Processing. Lawrence Erlbaum Associates.

Takashi Masuoka, editor. 1993. Nihongono Joken-Ilyogen (Conditional bipressions in Japanese). Kurosio Publishers, Tokyo. (iu Japancse).

Hirosato Nomura, 1992. Iinguistic analysis of law sentences. In Proceedings of the 6th International Symposium: Legal Knowledge and Legal Reasoning Systems, pages 10-18. Legal Expert System Association, October.

Marilyn Walker, Masayo lida, and Sharon Cote. 1990. Centering in Japanese discourse. In Proceedings of COIING 90. 